\title{
PPDPF cDNA Cloning from Two Bufo Species and Tissue Expression in B. gargarizans
}

\author{
Jiping Yue ${ }^{1}$, Guijin Jiang ${ }^{2}$, Xianyu Yang ${ }^{1 *}$, Huan Wang ${ }^{1}$, Lin Zhao ${ }^{1}$ \\ ${ }^{1}$ College of Animal Science and Technology, Zhejiang Agricultural and Forestry University, 666 Wusu Street, \\ Hangzhou, 311300, China \\ ${ }^{2}$ School of Forestry and Biotechnology, Zhejiang Agricultural and Forestry University, 666 Wusu Street, \\ Hangzhou, 311300, China
}

*Corresponding Author: Xianyu Yang, College of Animal Science and Technology, Zhejiang Agricultural and Forestry University, 666 Wusu Street, Hangzhou,311300, China, Email: yangxy78@zafu.edu.cn

\begin{abstract}
Cinobufacini injection was aqueous extract of toad (Bufo) dried skin, which has been widely used for cancer therapy in China. Recent studies indicated that the antitumor components included in cinobufacini are polypeptides. To identify these components, the skin plasmid cDNA library of Japanese toad (B. japonicus formosus) was screened. One of the transcripts is 864 base pair (bp) consisting of 144 bp 5 '-untranslated region (UTR), $384 \mathrm{bp}$ 3'-UTR and an open reading frame (ORF) of $336 \mathrm{bp}$ encoding a polypeptide of 111 amino acid residues. Homology analysis indicated $86 \%$ homolog with pancreatic progenitor cell differentiation and proliferation factor (PPDPF) of Xenopus laevis, which implicated in pancreas development. PPDPF ORF of Chinese toad (B. gargarizans) was cloned from its skin first strand cDNA encoding completely the same polypeptide as that of Japanese toad. Real-time quantitative polymerase chain reaction (qPCR) indicated PPDPF expressed in all organs of Chinese toad tested so far, while the highest expression is detected from pancreas. Considering the previous reports on the relevance of abnormal expression of PPDPF with several cancers including pancreatic cancer, PPDPF might be one of the crucial antitumor components of Bufo skin, so the successful cloning of PPDPF from Bufo skin is likely to shed light on some new antitumor drug development in future.
\end{abstract}

Keywords: Bufo japonicus formosus; Bufo gargarizans; PPDPF cloning; qPCR

\section{INTRODUCTION}

Amphibian skin and its secretions are rich of large amounts of biologically active compounds having great potential in new drug discovery ${ }^{[1-3]}$. In China, Bufo-origin materials such as toad skin (Chan'pi), cortex (Chan'yi), secretions (Chan'su) and its whole dried body (Gan-chan) were widely used in many prescriptions of traditional Chinese medicine for clinical treatments of many kinds of diseases, especially tumor control ${ }^{[4-7]}$. Cinobufocini injection, aqueous extract of toad dried skin, is a such kind of clinical drug especially aimed at advanced cancer ${ }^{[8]}$ including pancreatic cancer ${ }^{[9]}$. Recent studies indicated that peptides prepared from cinobufacini injection showed the same antitumor activity as that of the injection itself, which suggests that the polypeptides included in Bufo skin are the main active antitumor ingredients ${ }^{[10]}$. To analyze these polypeptides, the authors have been trying to clone cDNA from the skin cDNA plasmid library of Japanese toad (B. japonicus formosus) as well as from the skin first strand cDNA of Chinese toad (B. gargarizans) ${ }^{[11-13]}$. During these processes, the cDNA encoding pancreatic progenitor cell differentiation and proliferation factor (PPDPF) was obtained from both Japanese toad and Chinese toad.

$P P D P F$, also known as EXDPF (exocrine differentiation and proliferation factor) was first cloned from zebrafish, whose encoding protein has been reported as a decisive factor of the exocrine pancreas tissue development ${ }^{[14]}$. More interestingly, higher level of PPDPF ortholog has been detected in pancreatic cancer, breast cancer and kidney cancer ${ }^{[14]}$, indicating that super expression of PPDPF is likely to be involved in the pathogenesis of malignancy. Here, PPDPF is cloned from two Bufo species, whose expression has been detected in the organs of Chinese toad by qPCR (real-time quantitative polymerase chain reaction), which will be reported here. 


\section{Materials AND MethodS}

\subsection{Experimental Materials and Reagents}

Japanese toad skin plasmid cDNA library held by the Japan Advanced Industrial Science and Technology (AIST, Tsukuba, Japan) was authorized Zhejiang Agricultural and Forestry University (ZAFU) for research as part of a Material Transfer Agreement. Concerning this library construction, as reported previously ${ }^{[12]}$, pSD64TR (3250 base pairs) has been used as a vector, whose upstream primer is SP6 (5'-ATTTAGGTGACACTATAGAA-3') and the downstream one is S.D.A. (5'TTATGTAGCTTAGAGACTC-3'), and EcoR I and Xho I as cloning sites, and the length of the cDNAs ranged from 500 - 2000 base pairs (bp). The first strand cDNAs of Chinese toad skin were prepared from the individuals from the East lake Campus of ZAFU ${ }^{[11]}$.

The RNA extraction kit was purchased from Shanghai Bocai Biotechnology Company; pGM-T vector, Escherichia coli competent cells (DH5 $\alpha$ ) and Real Master Mix (SYBR Green) for qRT-PCR from Beijing Tiangen Biotechnology Limited Company; PCR kit (PrimeStar Max DNA polymerase) and PrimeScript ${ }^{\mathrm{TM}}$ II $1^{\text {st }}$ strand cDNA Synthesis kit from TaKaRa; and the primer synthesis and DNA sequencing were done by Shanghai Sang'ni Biotechnology Company.

\subsection{PPDPF cDNA Cloning of B. japonicus formosus and B. gargarizans}

cDNA cloning from Japanese toad was followed the method reported previously ${ }^{[12]}$. Briefly, Japanese toad skin plasmid cDNA library was transformed into E. coli (DH5 $\alpha$ ), and colony PCR was done using single colony suspension in LB medium as template, and XhoTT (5-AGATCTCTCGAGTTTTTTTTTTTT-3, a selfdesigned primer complementary with the area compassing the connection point of cDNA polyA tail and the downstream cloning site of Xho I) and SP6 as primers. Following this process, the recombinant plasmids were extracted and sequenced.

For the cloning of PPDPF ORF from Chinese toad, its dorsal skin total RNA was extracted and its first strand cDNAs were synthesized according to the manufacturer's protocol ${ }^{[11,12]}$. A set of primers was designed based on PPDPF cDNA sequence of Japanese toad (PPDPF-S: 5-CATCTTAAGTCCAAGCAAG-3; $P P D P F-R$ : 5-ATGCATGGAGGGATTAGG-3), which was also used for qPCR. Then PCR product was ligated into a pGM-T vector and sequenced with its primers of SP6 and T7 (5'TAATACGACTCACTATAGGG-3').

\subsection{Sequence Analysis and the Phosphorylation Site Prediction}

DNAstar/EditSeq was used to find out the ORF and deduce the amino acid sequence of the encoding protein. The potential phosphorylation sites of PPDPF were predicted via Net Phos 2.0 (http: //www.cbs.dtu.dk/services/NetPhos/).

\subsection{Homology Analysis of PPDPF Amino Acids}

Eleven PPDPF amino acid sequences of other animals downloaded by NCBI blast program (http://blast.ncbi.nlm.nih. gov/Blast.cgi) and PPDPF of Japanese toad and Chinese toad were aligned by MEGA 5.2, which was also used to construct the phylogenetic tree of PPDPF (bootstrap with 1000 replications).

\subsection{PPDPF Tissue Expression Analysis by qPCR}

Total RNA was extracted from each organ of B. gargarizans including brain, heart, intestines, kidney, liver, lung, oviduct, pancreas, skin, spleen, stomach and testis, and the first strand cDNA was synthesized as mentioned above. qPCR was performed for analysis of PPDPF expression in Chinese toad by Mx3000P (Stratagene, Agilent Technologies Inc., La Jolla, CA, USA). Here $\beta$-actin was used as a reference gene (upstream primer: 5-TTGAGACCTTCAACACC-3, downstream primer: 5-CTTGATGTCACGCACAA-3). Three sets of the first strand cDNA of each organ from 3 individuals were used. Data was analyzed by MxPro Comparative Quantitation and MS excel.

\section{RESUlTS}

\subsection{PPDPF Cloning of B. japonicus formosus and B. gargarizans}

Sequencing analysis indicated a transcript of $864 \mathrm{bp}$ consisting of $144 \mathrm{bp} 5$ '-untranslated region (UTR), $384 \mathrm{bp}$ 3'-UTR and an ORF of $336 \mathrm{bp}$, whose encoding polypeptide is consisting of 111 amino acid 
residues (Fig. 1) with high homology with PPDPF of other animals. This transcript has been deposited into GenBank and named as B. japonicus formosus PPDPF with an accession number of KJ128292.

From the skin first strand cDNAs of Chinese toad, 44 clones were obtained. Twenty-six out of them showed the same ORF of $336 \mathrm{bp}$ with only one nucleotide difference from the ORF of Japanese toad $P P D P F$ (Fig. 1).

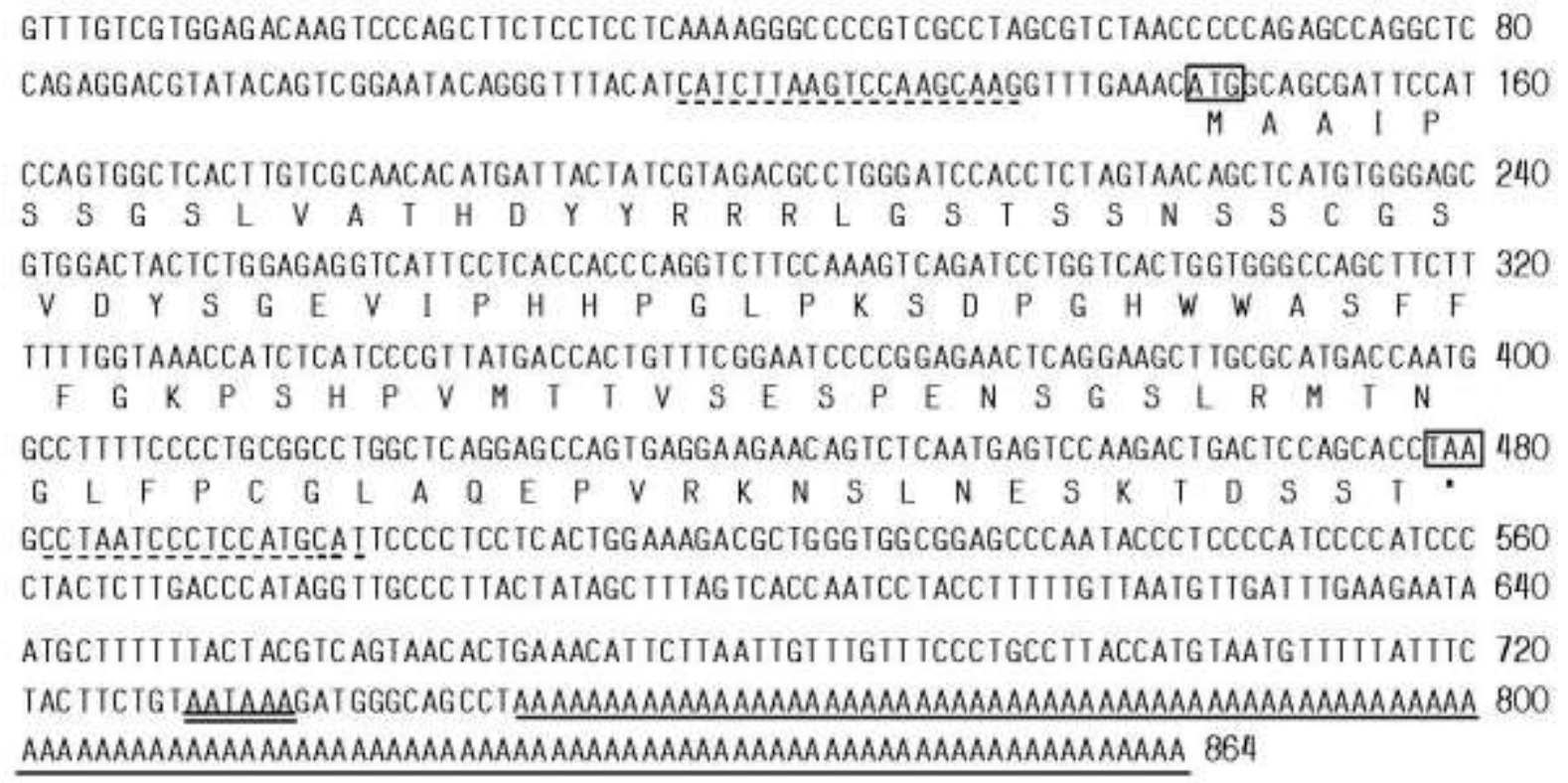

Figure 1 PPDPF cDNA and its deduced amino acid sequence of Bufo japonicus formosus

$\square$ : Start and stop codons; Nucleotide in shadow: Substituted by "T" in Chinese Bufo; _ : Poly (A) tail; ==: Polyadenylation signal; .....: Primers of PPDPF-S and PPDPF-R.

Due to this nucleotide substitution did not result in the change of its encoding amino acid, the encoding polypeptide was completely the same as PPDPF of Japanese toad. The cDNA clone appeared in high frequency has been deposited into GenBank (accession number: KJ128291) and named as B.gargarizans $P P D P F$. Concerning the remaining 18 clones, they showed 1 or more nucleotide difference and were different from each other indicating the possible artificial introduction of nucleotide substitution during the experimental processes in vitro including PCR (data not shown).

\subsection{Phosphorylation Site Prediction of Bufo PPDPF}

Thirteen potential phosphorylation sites (Ser9, Ser23, Ser25, Ser28, Ser29, Ser36, Ser74, Ser101, Ser105, Ser109, Ser110, Tyr69, and Tyr70) were found in PPDPF of two Bufo species, which suggests that the function of PPDPF might be regulated by the upstream factors (Table 1).

Table1. Potential phosphorylation sites (shade) of PPDPF two Bufo species

\begin{tabular}{|l|l|l|l|l|l|}
\hline Position & Context & Score & Position & Context & Score \\
\hline 9 & PSSGSAT & 0.629 & 70 & PIMTTVSES & 0.789 \\
\hline 23 & RRLGSTSSN & 0.990 & 74 & TVSESPENS & 0.998 \\
\hline 25 & LGSTSSNSS & 0.764 & 101 & VRKNSLNES & 0.995 \\
\hline 28 & TSSNSSCGS & 0.970 & 105 & SLNESKIDS & 0.991 \\
\hline 29 & SSNSSCGSV & 0.944 & 109 & SKTDSST & 0.538 \\
\hline 36 & SVDYSGEVI & 0.966 & 110 & KTDSST & 0.690 \\
\hline 69 & HPIMTTVSE & 0.898 & & & \\
\hline
\end{tabular}

\subsection{Homology Analysis of PPDPF}

PPDPF homology analysis of two Bufo species indicated 86\% homology with Xenopus laevis, $65 \%$ with Homo sapiens, and 59\%-80\% among 9 other animals (Fig. 2). 

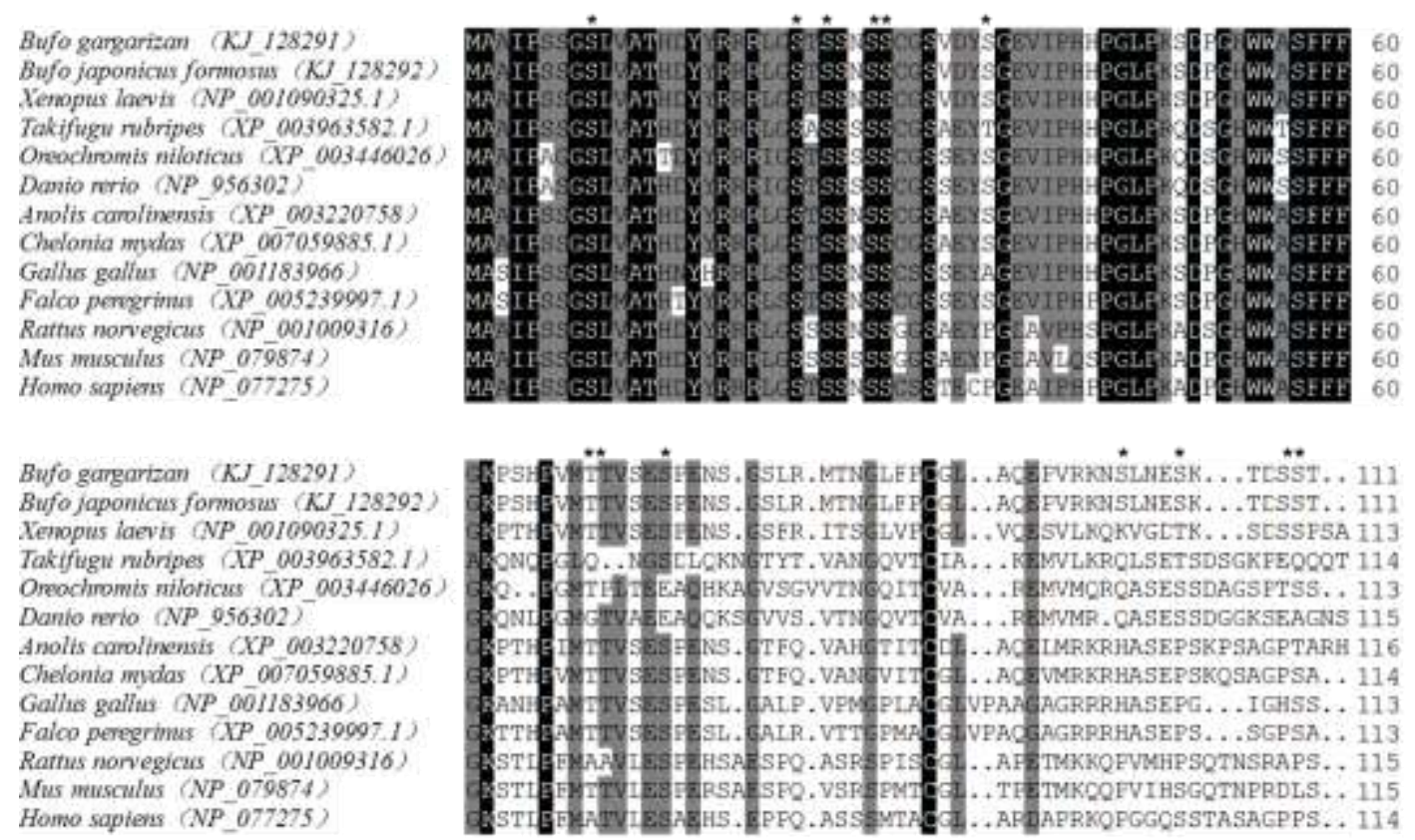

Figure 2 Multiple amino acid sequence alignment of PPDPF of Bufo with that of 11 different animals

100\% homology; $\square$ : Above 75\% homology; $\square$ : Homology less than 75\%. *: Potential phosphorylation sites of Bufo PPDPF.

Based on the phylogenetic tree (Fig. 3), 3 fish (Takifugu rubripes, Oreochromis niloticus, Danio rerio), 3 amphibian species (X. laevis as well as two Bufo species), 2 reptiles (Anolis carolinensis, Chelonia mydas), 2 birds (Gallus gallus, Falco peregrinus), and 3 mammals (Homo sapiens, Mus musculus, Rattus norvegicus) gathered in one branch, respectively, which is consistent with the traditional animal taxonomy. Among 13 potential phosphorylation sites of Bufo, the first five were highly conserved among these 13 animals (Fig. 2).

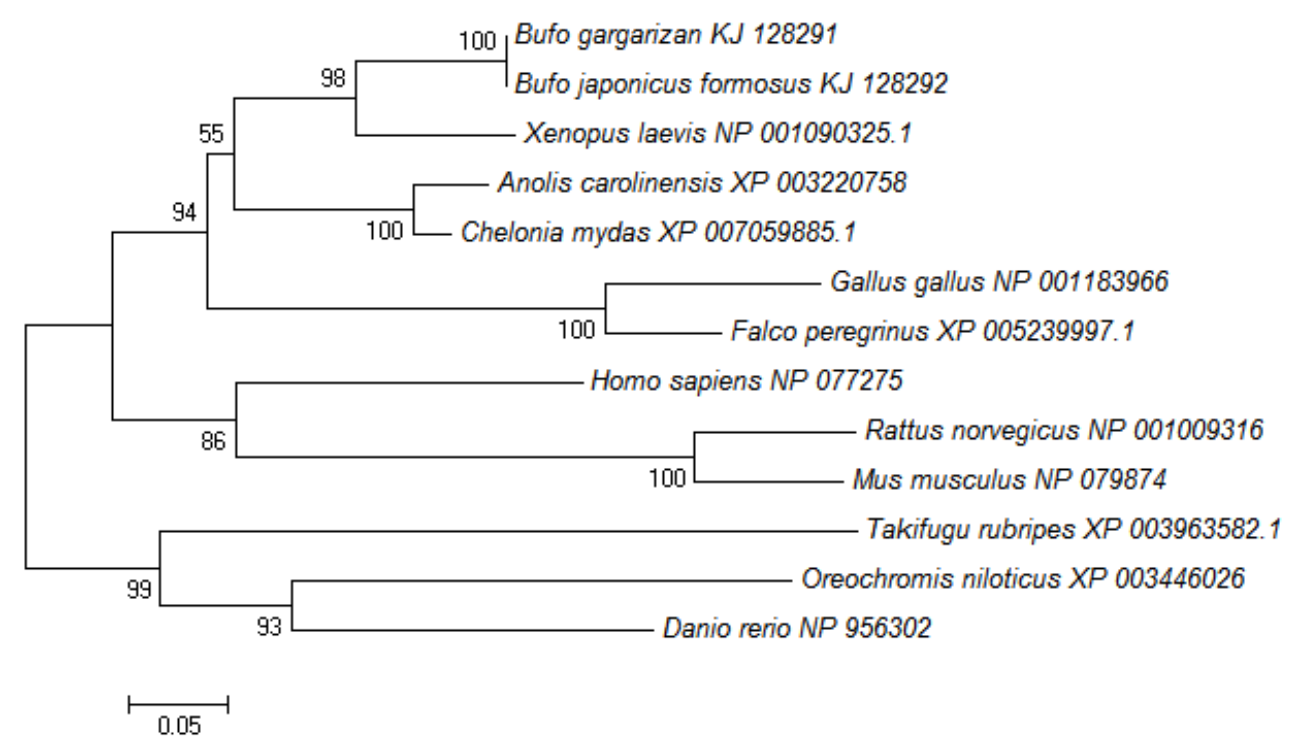

Figure 3 Phylogenetic tree of PPDPF constructed by MEGA 5.2

The scale bar represents the number of changes per amino acid position.

\subsection{PPDPF Expression Analysis in Different Organs of Bufo gargarizans by qPCR}

qPCR analysis of B. gargarizans in the brain, heart, lung, liver, pancreas stomach, intestines, kidney, testis, oviduct, spleen and skin showed that PPDPF was expressed in all these organs, and the expression level was the highest in pancreas (Fig. 4). 


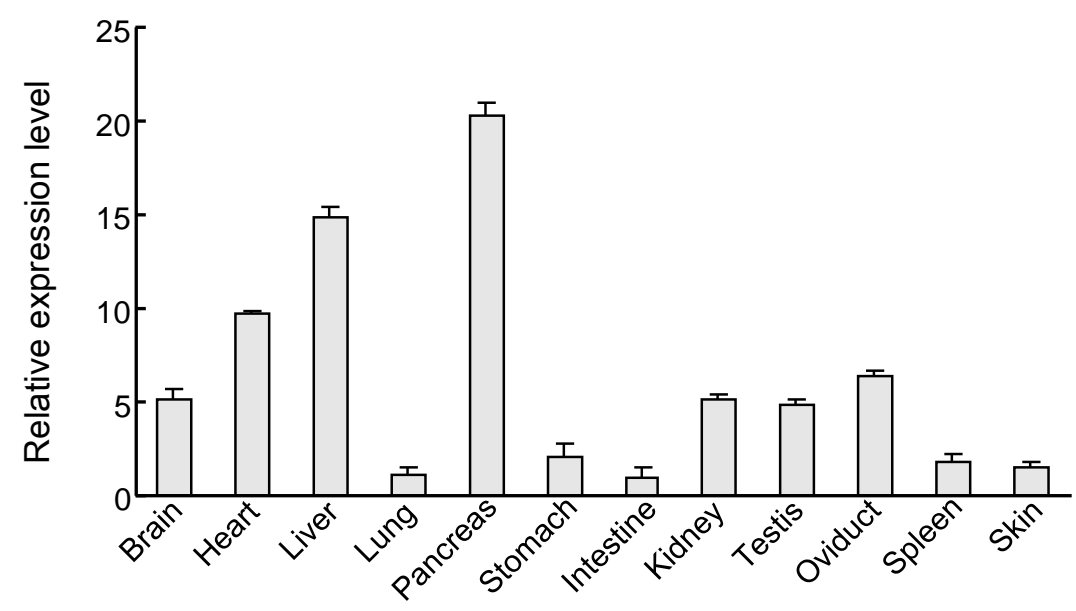

Figure 4 PPDPF expression in different organs of Bufo gargarizans

Abscissa represents the organs of Bufo gargarizans being tested, ordinate represents relative expression quantity relative to $\beta$-actin. Error bars show square deviation of each organ from 3 individuals.

\section{DISCUSSION}

So far, there are many reports concerning on the pharmaceutical functions of Bufo-skin and its secretions $[3,15,16]$. In recent years, several reports clearly indicated the antitumor effects of the polypeptides extracted from cinobufacini injection ${ }^{[9,17]}$ which comes from toad skin. Therefore, it is both essential and urgent to identify these polypeptides. Authors have been screening/cloning the cDNA form the skin plasmid cDNA library of Japanese toad and the skin first strand cDNA of Chinese toad to contribute such studies ${ }^{[11-13]}$, trying to seek out related factors.

In current study, PPDPF cDNA was successfully cloned from skin of Japanese toad and Chinese toad both encoding the same polypeptide consisting of 111 amino acid residues (Fig. 1) having high homology with that of other vertebrates (Fig. 2,3). Many potential phosphorylation sites indicate PPDPF function might be upstream regulated (Table 1). qPCR showed PPDPF expressed in all the tested organs of Chinese toad (Fig. 4) indicating the therapeutic values of the whole body of Bufo but not limited to its skin.

Despite the fewer studies of $P P D P F / E X D P F$ so far, it's certainly a decisive factor of the exocrine pancreas tissue development due to the PPDPF knocking down leading to cell cycle arrests through upregulation of $\mathrm{p} 21^{\mathrm{Cip}}, \mathrm{p} 27^{\mathrm{Kip}}$ and a severe reduction of the cell number of exocrine progenitor by $70 \%$; however, the overexpression of $P P D P F$ led to increased exocrine size indicating its involvement in the pancreatic development in Danio rerio ${ }^{[14]}$.

Pancreatic cancer is one of the leading causes of cancer deaths as it's often highly aggressive and resistant to treatments available at the time of diagnosis ${ }^{[18]}$. In addition, most malignant pancreatic tumors are derived from exocrine portion ${ }^{[19]}$. Interestingly, the EST (Expressed Sequence Tag) expression profile showed higher level of PPDPF ortholog has been detected in several cancers including pancreatic cancer, breast cancer and kidney cancer, indicating that super expression of PPDPF is likely to be involved in the pathogenesis of malignancy ${ }^{[14]}$, suggesting the possible pivotal role towards cancer. In a word, the current study will lay foundation for exploring new antitumor drug to control cancer.

\section{ACKNOWLEDGEMENT}

This work was partially supported by the Grant from National Natural Science Foundation of China (No. 31772409, 31372149).

\section{REFERENCES}

[1] Clarke BT. The natural history of amphibian skin secretions, their normal functioning and potential medical applications. Biol Rev Camb Philos Soc 1997, 72(03): 365-379.

[2] Novković M, Simunić J, Bojović V, Tossi A, Juretić D. DADP: the database of anuran defense peptides. BMC 2012, 28(10): 1406-1407.

[3] Qi F, Li A, Inagaki Y, Kokudo N, Tamura S, Nakata M, Tang W. Antitumor activity of extracts and 
compounds from the skin of the toad Bufo bufo gargarizans cantor. Int Immunopharmacol 2011, 11(3): 342349.

[4] Enomoto A, Rho MC, Komiyama K, Hayashi M. Inhibitory effects of bufadienolides on interleukin-6 in MH-60 cells. J Nat Prod 2004, 67(12): 2070-2072.

[5] Hong Z, Chan K, Yeung H. Simultaneous determination of bufadienolides in the traditional Chinese medicine preparation, Liu-Shen-Wan, by liquid chromatography. J Pharm Pharmacol 1992, 44(12): 10231026.

[6] Li C, Hashimi SM, Cao S, Mellick AS, Duan W, Good D. The mechanisms of Chansu in inducing efficient apoptosis in colon cancer cells. Evid Based Complement Alternat Med 2013, 2013: 84905.

[7] Qin TJ, Zhao XH, Yun J, Zhang LX, Ruan ZP, Pan BR. Efficacy and safety of gemcitabine-oxaliplatin combined with huachansu in patients with advanced gallbladder carcinoma. World J Gastroenterol 2008, 14(33): 5210-5216.

[8] Gomes A, Bhattacharjee P, Mishra R, Biswas AK, Dasgupta SC, Giri B. Anticancer potential of animal venoms and toxins. Indian J Exp Biol 2010, 48(2): 93-103.

[9] Yin JH, Zhu XY, Shi WD, Liu LM. Huachansu injection inhibits metastasis of pancreatic cancer in mice model of human tumor xenograft. BMC Complement Altern Med 2014, 14(1): 483.

[10] Wu X, Gao B, Yang J, Bian BL, Wang HJ. In vitro anti-proliferation effect of peptides from cinobufacini injection. Acta Pharmaceutica Sinica 2012, 47(6): 822-826.

[11] Hu QL, Zhang SF, Yang XY, Yu MH, and Zhuge H. Bufo gargarizans mcl-1 cloning and its prokaryotic recombinant protein expression. Acta Pharmaceutica Sinica 2013, 48(10): 1624-1628.

[12] Ji YC, Zhuge H, Zhang SS, Zhang SF, and Yang XY. Cloning the sterol carrier protein 2 genes of Japanese toad (Bufo japonicus formosus) and Chinese toad (Bufo gargarizans) and its tissue expression analysis. Zoological Research 2014, 35(5): 398-403.

[13] Zhuge H, Yuan JQ, Zhang SF, Yang XY. Cloning and bioinformatic analysis of TAGLN2 cDNA of Bufo japonicus formosus. Acta Pharmaceutica Sinica 2013, 48(2): 250-254.

[14] Jiang Z, Song JB, Qi F, Xiao A, An XZ, Liu NA. Exdpf is a key regulator of exocrine pancreas development controlled by retinoic acid and ptf1a in zebrafish. PLoS One 2008, 6(11): e293.

[15] Wang DL, Qi FH, Tang W, Wang FS. Chemical constituents and bioactivities of the skin of Bufo bufo gargarizans cantor. Chem biodiversity 2011, 8(4): 559-567.

[16] Wang L, Raju U, Milas L, Molkentine D, Zhang Z, Yang P. Huachansu, containing cardiac glycosides, enhances radiosensitivity of human lung cancer cells. Anticancer Res 2011, 31(6): 2141-2148.

[17] Qi J, Tan C, Hashimi S, Zulfiker A, Good D, Wei M. Toad glandular secretions and skin extractions as antiInflammatory and anticancer agents. Evid Based Complement Alternat Med 2013, 2014: 312684.

[18] Niederhuber JE, Brennan MF, Menck HR. The national cancer data base report on pancreatic cancer. Cancer 1995, 76(9): 1671-177.

[19] Cowgill SM, Muscarella P. The genetics of pancreatic cancer. Am J Surg 2003, 186(3): 279-286.

Citation: Jiping Yue. et.al., "PPDPF cDNA Cloning from Two Bufo Species and Tissue Expression in B. gargarizans", International Journal of Research Studies in Biosciences (IJRSB), vol. 6, no. 4, pp. 35-40, 2018. http://dx.doi.org/10.20431/2349-0365.0604005

Copyright: (C) 2018 Authors. This is an open-access article distributed under the terms of the Creative Commons Attribution License, which permits unrestricted use, distribution, and reproduction in any medium, provided the original author and source are credited. 